POLLACK PERIODICA

An International Journal for Engineering and Information Sciences

DOI: $10.1556 / 606.2018 .13 .1 .20$

Vol. 13, No. 1, pp. 225-236 (2018)

www.akademiai.com

\title{
SPECIAL HYPERCUBE MODELS AND 3D-TESSELLATIONS BASED ON FIVE CUBES JOINING THE VERTICES OF THE PLATONIC DODECAHEDRON
}

\author{
Dedicated to the memory of Professor Slavik Jablan, \\ a mathematician and outstanding teacher of artistic geometry
}

\author{
László VÖRÖS \\ Department of Architectural and Visual Studies, Faculty of Engineering and Information \\ Technology, University of Pécs, Boszorkány út 2, H-7624 Pécs, Hungary \\ e-mail: vorosl@mik.pte.hu
}

Received 14 July 2017; accepted 18 September 2017

\begin{abstract}
The 3-dimensional model of any $k$-dimensional cube can be constructed by starting $k$ edges whose Minkowski sum can be called zonotope. Combined $2<j<k$ initial edges result in 3 -models of $j$-cubes as parts of a $k$-cube. Suitable combinations of these zonotopes result in 3 dimensional space-filling mosaics. The base of the described cases, presented here, is five cubes constructed with joining vertices in the Platonic dodecahedron. These have 15 differently directed edges whose above zonotope is the 3-model of the 15-cube, or the Archimedean truncated icosidodecahedron. The reported further zonotopes are 3-models of lower-dimensional parts of this one. Pedagogical aspects of this topic are also emphasized.
\end{abstract}

Keywords: Platonic and Archimedean solid, Hypercube model, Spatial and planar tessellations

\section{Introduction}

\subsection{Specific zonotope models of hyper cubes}

This paper is strongly based on the former investigations of the author, especially on [1] of this Periodica and on [2]. The animations in [3] can give some visual supports, a transfer of 3-mosaics in the analogue of higher-dimensional geometry.

Lifting the vertices of a $k$-sided regular polygon from their plane, perpendicularly to the same height, and joining them with the center of the polygon, there are given $k$ initial edges of the $k$-dimensional cube (further on $k$-cube) modeled in the threedimensional space (3-model). From these, the 3-models or their polyhedral surface 
(Fig. 1, left) can also be generated by the well-known procedure. That is, by moving the lower-dimensional elements along edges parallel to the direction of the next dimension [1]. Thus each polyhedron will become a zonotope [4], [5] i.e. a 'translational sum' of some segments (Minkowski sum) [6].

Suitable combinations of the above zonotopes can result in 3-dimensional spacefilling mosaics. The investigated periodical tessellations always contain the 3 -model of the $k$-cube and of necessary $j$-cubes derived from it, constructed with combined $2<j<k$ initial edges (Fig. 1, right) [1]. Moving planes, intersecting the mosaics, create series of tessellations or grid-patterns transforming into each other. These can be presented in various animations [3].

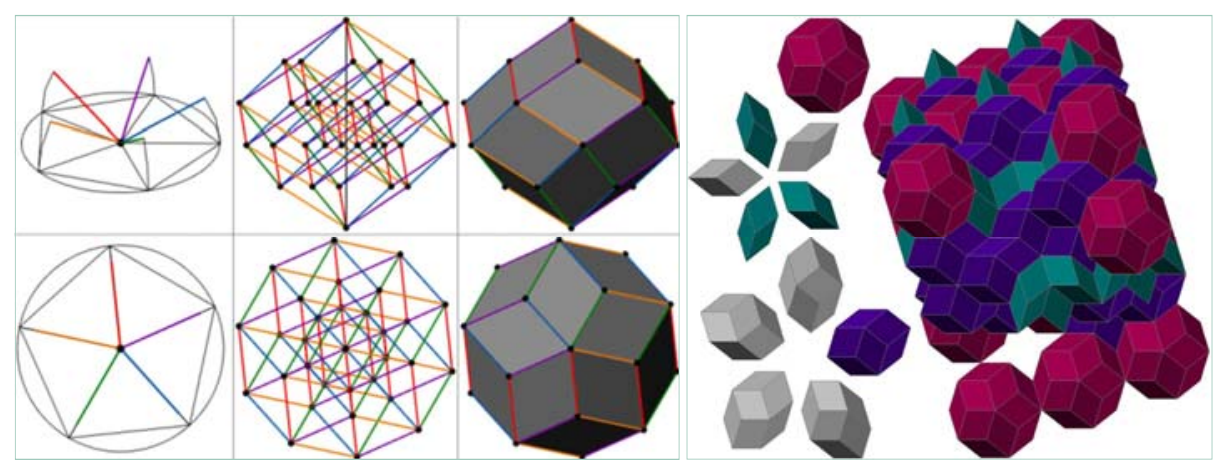

Fig. 1. The above described 3-model of the 5-cube and a space-filling mosaic based on this

\subsection{Three-dimensional Platonic and Archimedean solids related to zonotope models of hyper cubes}

The edges of the next considered polyhedra have 6 different spatial directions: Platonic tetrahedron $(3,3,3)$ and octahedron $(3,3,3,3)$ as well as the Archimedean truncated tetrahedron $(3,6,6)$, cuboctahedron $(3,4,3,4)$ and truncated octahedron $(4,6,6)$. The here used type of vertex figure (based on the Schläfli symbol) enumerates the regular polygons meeting in each vertex of a polyhedron in the given order. A 3-model of the 6-cube can be constructed with the former 6 edges. The hull (zonohedron) of this is the Archimedean truncated octahedron. This is a Voronoi cell that can fill the 3-space [6].

The edges of the next polyhedra have 9 different spatial directions: Archimedean truncated cube $(3,8,8)$, (small) rhombicuboctahedron $(3,4,4,4)$ and great rhombicuboctahedron or truncated cuboctahedron $(4,6,8)$. The same set of basic edges can be gained from Platonic solids if a tetrahedron (or the dual pair of two ones) is constructed into a cube or the dual pair of the octahedron and the cube is considered. A 3-model of the 9-cube can be built with the former 9 edges. The hull (zonohedron) of the whole 3 model is the Archimedean truncated cuboctahedron (4,6,8). The elements of the above 3 -model of the 6-cube join the parts of the 3-model of the 9-cube. The Archimedean truncated octahedron and the Platonic hexahedron are 3-models of lower-dimensional parts (6-cube and 3-cube) of the 9-cube modeled with the Archimedean truncated 
cuboctahedron. The edges of these derived parts have the directions of the 9 initial edges of the 9-cube model [6].

There are periodical space-filling mosaics built with the above solids. The repeated parts of these belong to the 28 convex uniform honeycombs enumerated by Branko Grünbaum [7]. The vertices of the stones in the above mosaics join a point net structure [2].

The edges of the next polyhedra have 15 different spatial directions: Platonic dodecahedron $(5,5,5)$ and icosahedron $(3,3,3,3,3)$ as well as the Archimedean icosidodecahedron $(3,5,3,5)$, truncated dodecahedron $(3,10,10)$, truncated icosahedron $(5,6,6)$, rhombicosidodecahedron $(3,4,5,4)$ and truncated icosidodecahedron $(4,6,10)$. These are the initial edges of a 3-model of the 15-cube (Fig. 2, right). The hull (zonohedron) of this model is the Archimedean truncated icosidodecahedron (Fig. 3, right).

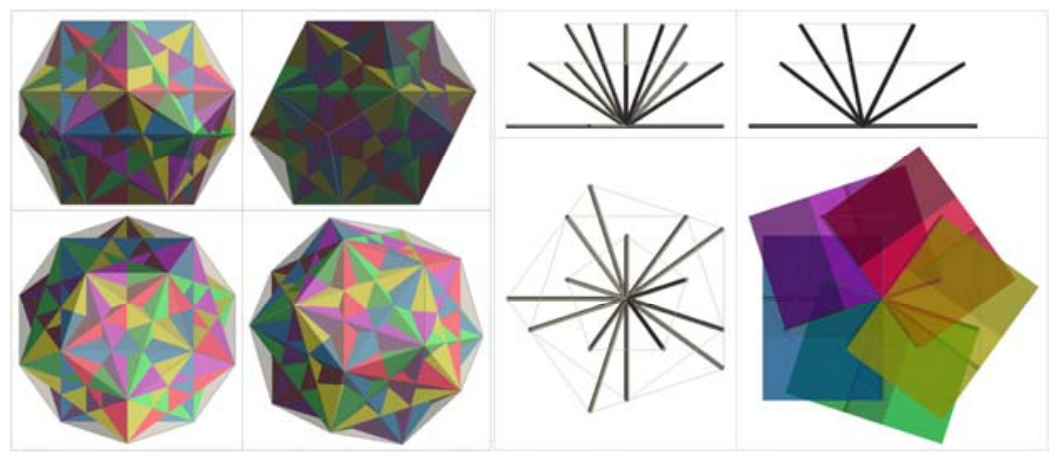

Fig. 2. Five cubes constructed into the Platonic dodecahedron, orthographic views and axonometric projection. Their copies have a common vertex and 15 differently directed edges
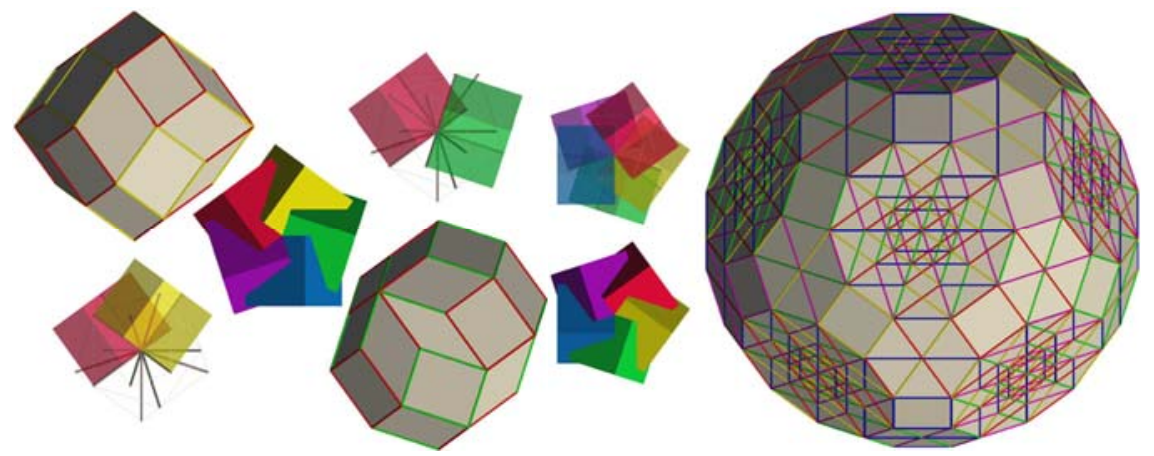

Fig. 3. The edges of any pairs of the cubes are parallel to edges of congruent 3-models of the 6-cube, and edges of the 5 cubes are parallel to the edges of the 3-model of the 15-cube

Is it possible to construct a periodical tessellation based on the Archimedean truncated icosidodecahedron? It has tenfold rotational symmetry around 6 axes. It is no part of any convex uniform honeycombs. Only the Platonic and Archimedean 
polyhedra, showed above, have parallel edge set to this polyhedron. However these polyhedra are not zonohedra except $(4,4,4),(4,6,6)$ and $(4,6,8)$. That means these cannot be 3-models of lower-dimensional parts of the 15-cube.

The normal cube and other right and oblique prisms with regular polygon bases can be 3-models of lower-dimensional parts of this 3-model of the 15-cube if the number of the base polygon edges is even and all edges are parallel to the edges of the truncated icosidodecahedron. This is the only correspondence to the convex uniform honeycombs.

The only way to find the suitable 3-model set of the lower-dimensional parts added to the considered 3-model of the 15-cube, is then the arrangement of this stones creating a periodical space-filling mosaic.

After this overview, which will be continued at the concluding part shortly as well, the next part of the paper concentrates on the title problem in more details.

\section{Hypercube models and tessellations based on the truncated icosidodecahedron $(4,6,10)$}

The bases of the present cases are five cubes. Their vertices join those of the Platonic dodecahedron. The edges of these cubes are diagonals of the pentagonal faces and are parallel to the edges of the dodecahedron (Fig. 2, left). The above 5 cubes, shifted parallel, have a common vertex and 15 differently directed edges (Fig. 2, right).

\subsection{The general method}

The edges of any pairs, triplets and quartets of the cubes are parallel to edges of congruent 3-models of the 6D, 9D and 12D cubes. The case of the 6-cube is showed in Fig. 3, left. The edges of the 5 cubes are parallel to the edges of a 3-model of the 15-cube. The hull (zonohedron) of this is the truncated icosidodecahedron (Fig. 3, right) since the cube edges are parallel to the dodecahedron edges. The above zonotopes (zonohedra) model the lower-dimensional $j$-cubes of the 15-cube.

The specified orientation of the above mentioned 3-models of the 6-, 9-, 12-, 15 -cubes simplifies the symmetry properties in top view. Two examples of these reorientations are showed in Fig. 4. This method helps to construct periodical spacefilling mosaics with these initial elements. The stone sets of these mosaics can be considered as subsets of the derived elements of the 15-cube model. The honeycombs of these tessellations have several symmetry planes and the construction of the mosaics progressively prepares the periodical space-filling tessellation based on the truncated icosidodecahedron, as desired.

It can be mentioned here that the common part of the five cubes, constructed into the dodecahedron, is a regular rhombic triacontahedron. It is a possible 3-model of the 6 -cube if the inner edges are constructed parallel to the outer ones. More about the particular properties of this model has been published in [8]. A periodic spatial mosaic is shown in Fig. 5. That is constructed with the rhombic triacontahedron as 3-model of the 6-dimensional cube and with the derived lower-dimensional cubes' models. The animation of cut planar tessellations can be seen at [3]. 


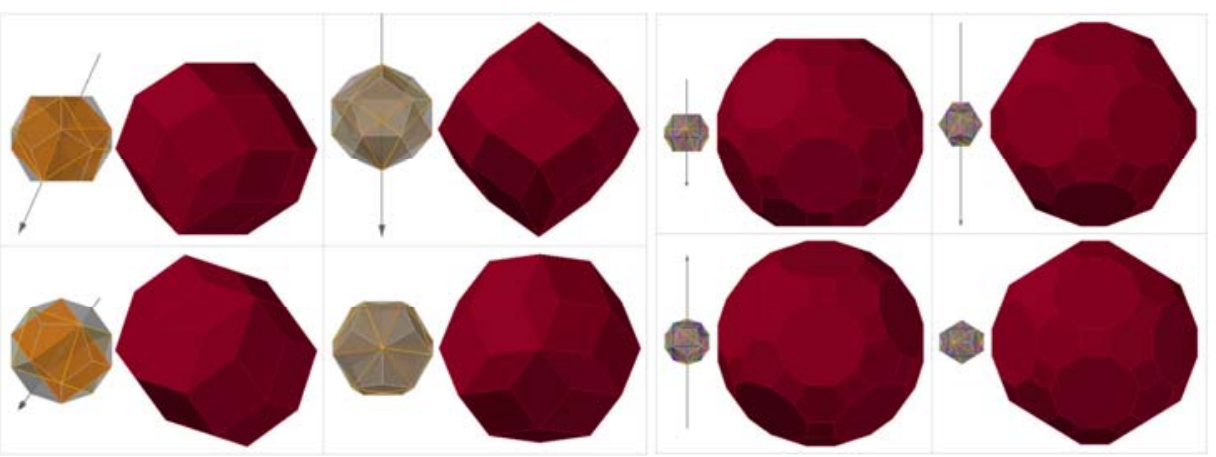

Fig. 4. The specified orientation of the 3-model of the 6-cube (left) and the 15-cube (right) in front and top views

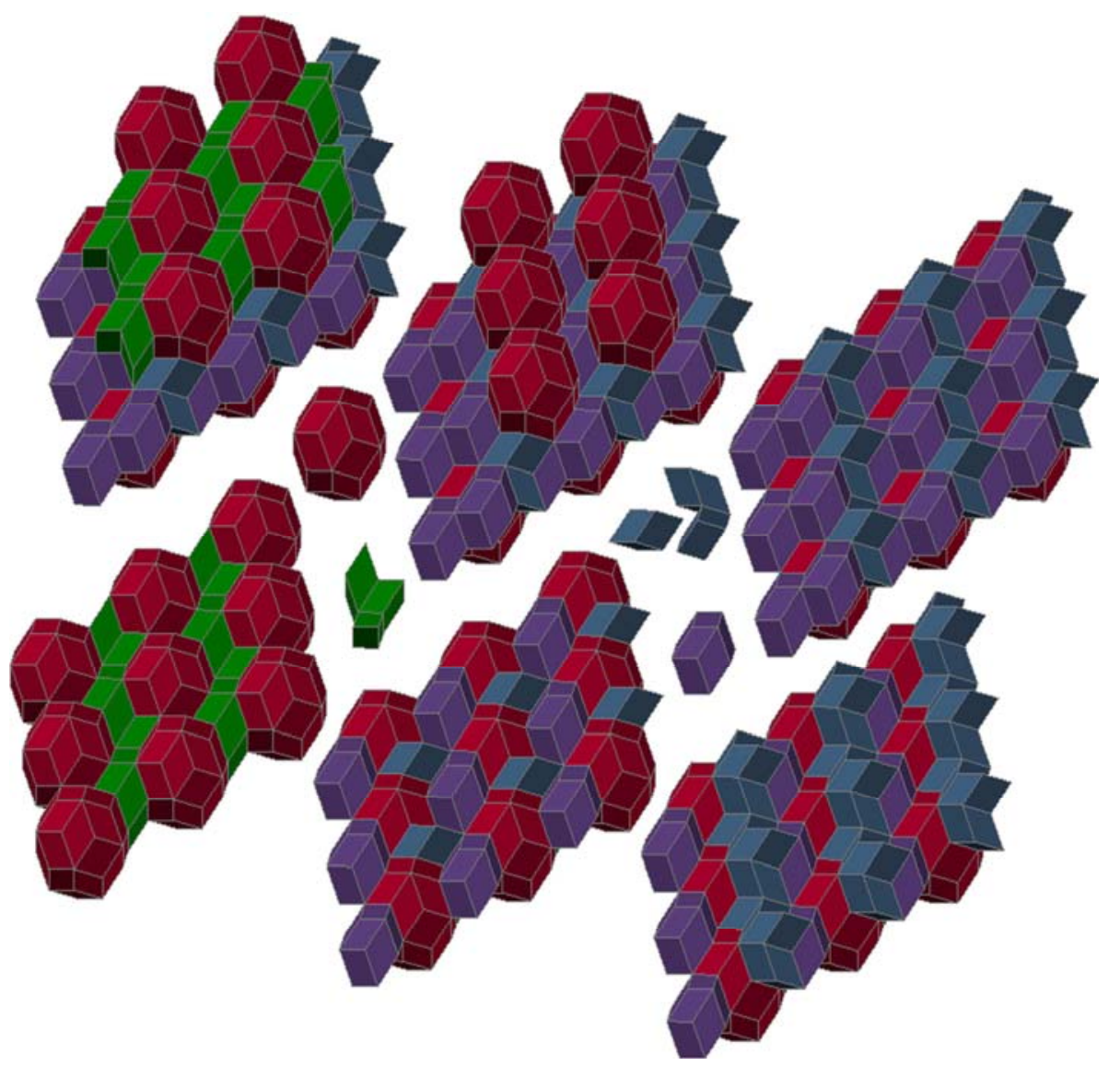

Fig. 5. Periodic spatial mosaic based on the rhombic triacontahedron model of the 6- cube (Built from the bottom left part, counter clockwise.) 
The process of the mosaic construction and the series of cut planar tessellations are similar in all the above cases: by using elements derived from the truncated icosidodecahedron. It is presented with the 15-cube in a picture series, showing some elements of the spatial periodical mosaic, in Fig. 6 and Fig 7. The main structure can be characterized by the 3-models of the 15-cubes arranged in horizontal hexagonal net. The planes intersecting the mosaics need special arrangement as it can be seen in Fig. 8 .

Animations of the planar tessellations related to the 6-, 9-, 12, -15-cubes can be seen at [3]. There are showed two examples in case of the 9-cube as well, created from two different mosaics.
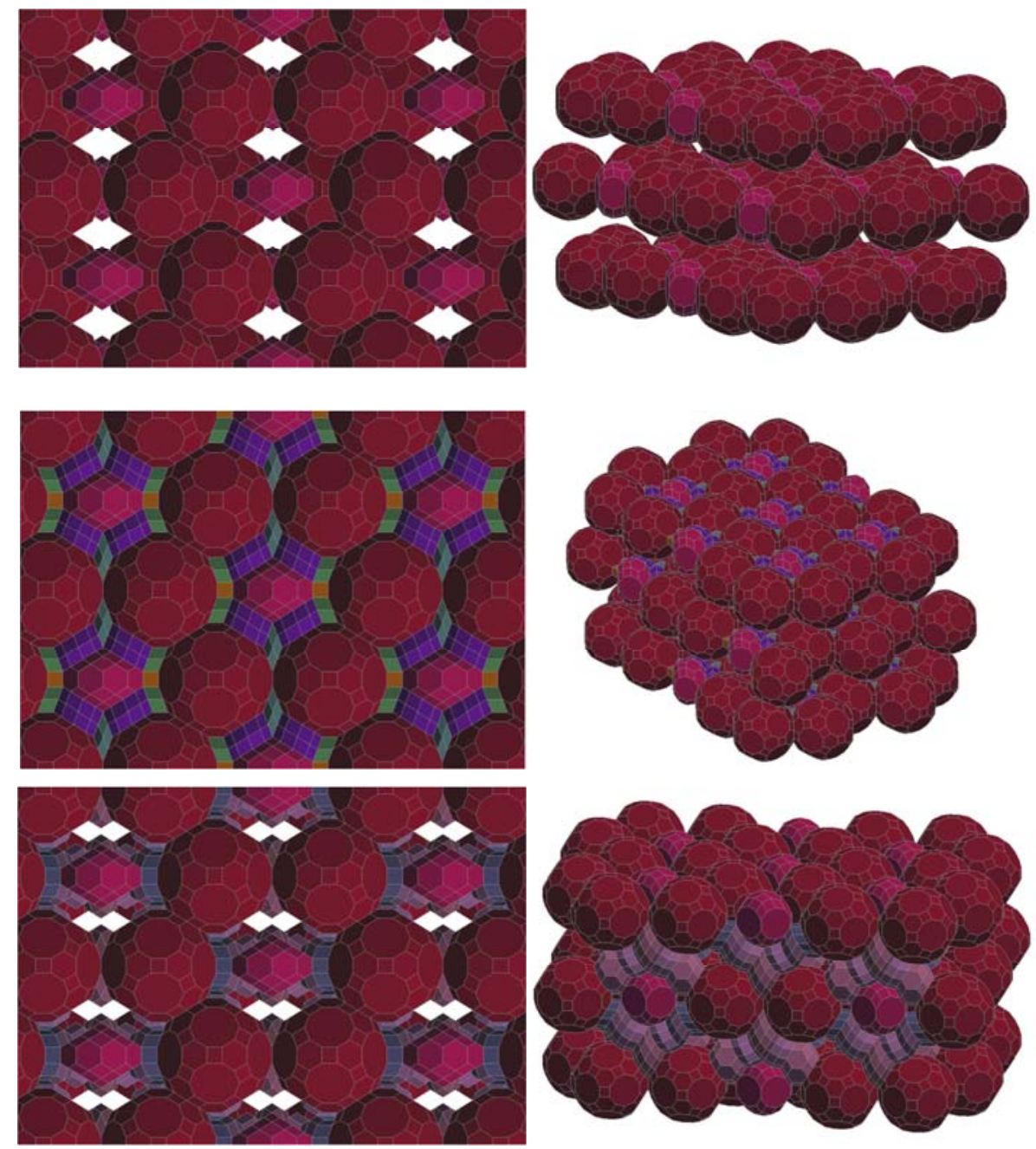

Fig. 6. Different sets of mosaic stones in the tessellation based on the 3-model of the 15-cube in top view (left) and in axonometric projection (right)

Pollack Periodica 13, 2018, 1 

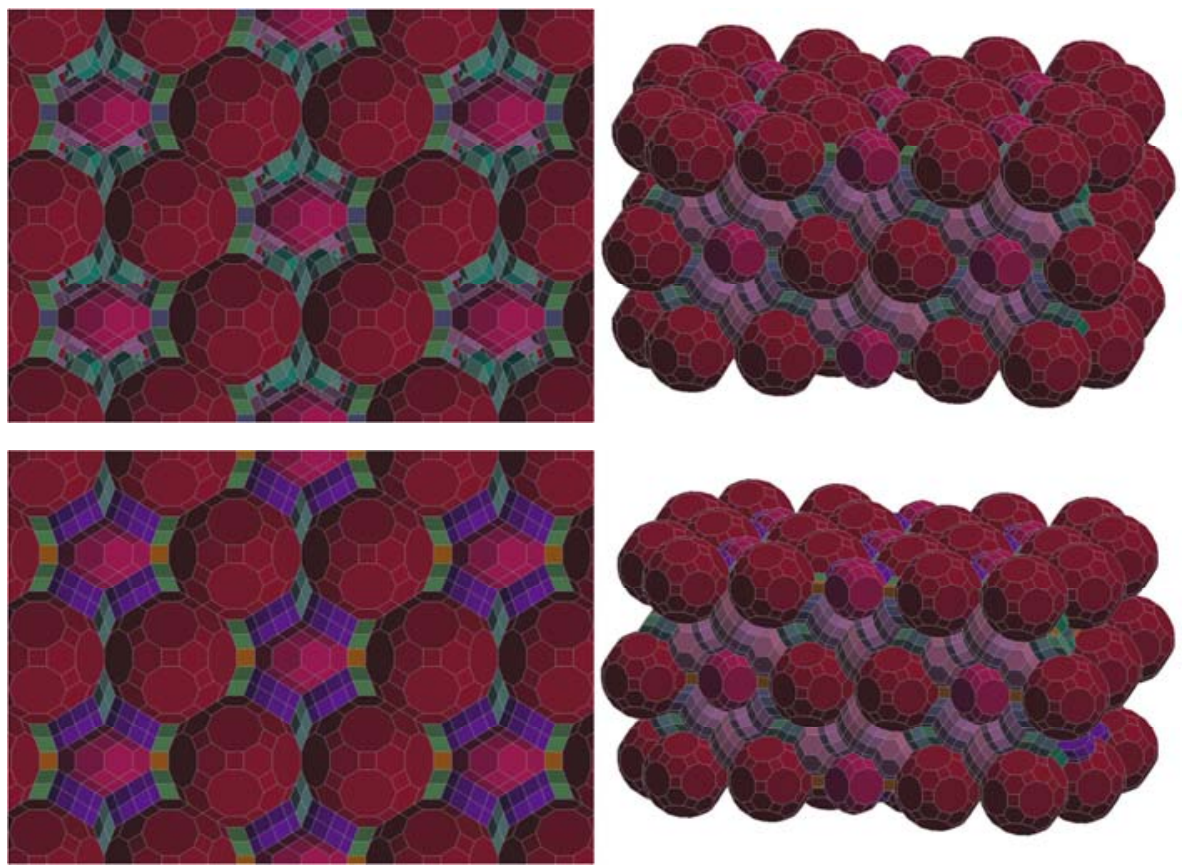

Fig. 7. Different sets of mosaic stones in the tessellation based on the 3-model of the 15-cube in top view (left) and in axonometric projection (right).

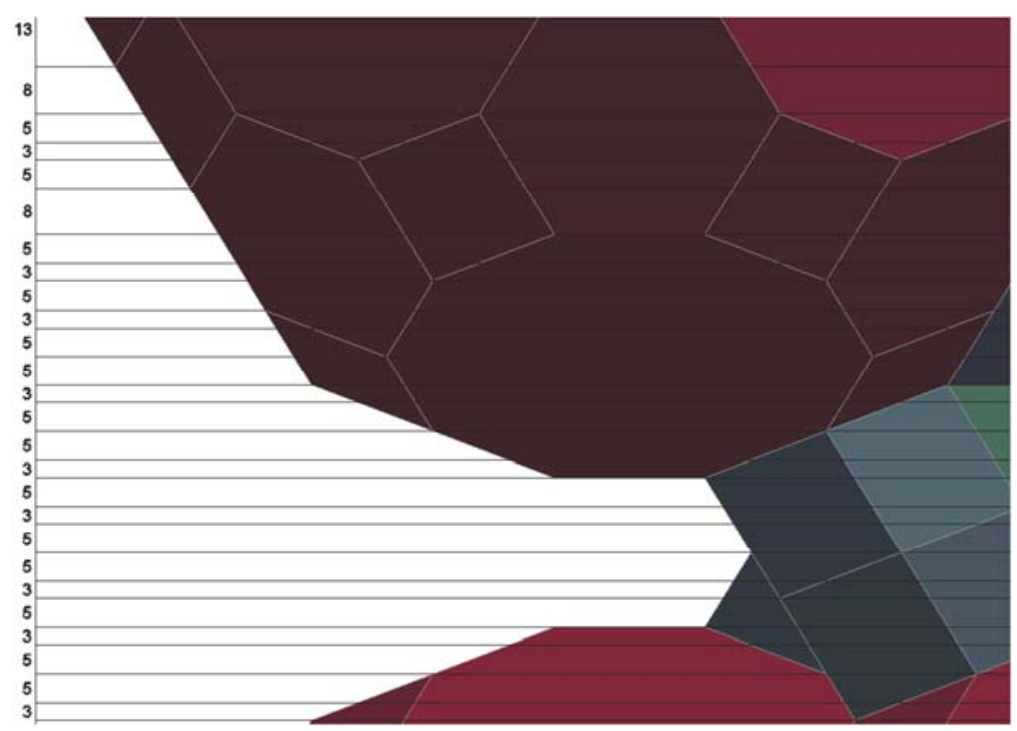

Fig. 8. The vertices of the stones join horizontal planes. The vertical distances of these are proportioned to each other by the golden mean. Thus we need the Fibonacci numbers to define further planes for the cut and in animation queued horizontal tessellations 


\subsection{A possible specific way of modeling}

Orthogonal triplets of the initial edges of modeled higher-dimensional cubes can be parallel to the cube edges constructed in the Platonic dodecahedron. The first cube, created by the help of one of these triplets, is considered as the model of a 3dimensional part of the specific hypercube. The number of the edges is a multiple of three. The first 3-cube can be shifted in direction of an edge of the second one. A 3model of the 4-cube is done (Fig. 9, left). Shift in direction of the second differently oriented edge of the second cube gives a 3-model of the 5-cube (Fig. 9, right). Shift in the direction of the third, differently oriented edge of the second cube results in the 3 model of the 6-cube (Fig. 10, left).

The edges of shifts, parallel to three differently oriented edges of the second cube, are also shifted, so these define the new, yellow cubes (Fig. 10, right). All cubes will be shifted along edges parallel to the third differently oriented cube, and so on. The process repeats continuously the same three steps.

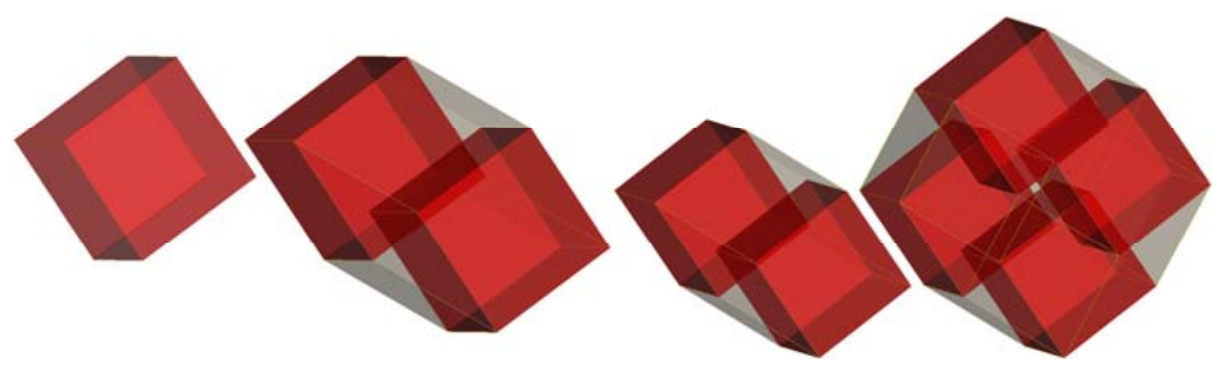

Fig. 9. The chosen first cube is shifted in direction of an edge of the second cube: 3D-4D. Shift in direction of the second edge of the second cube: 4D-5D
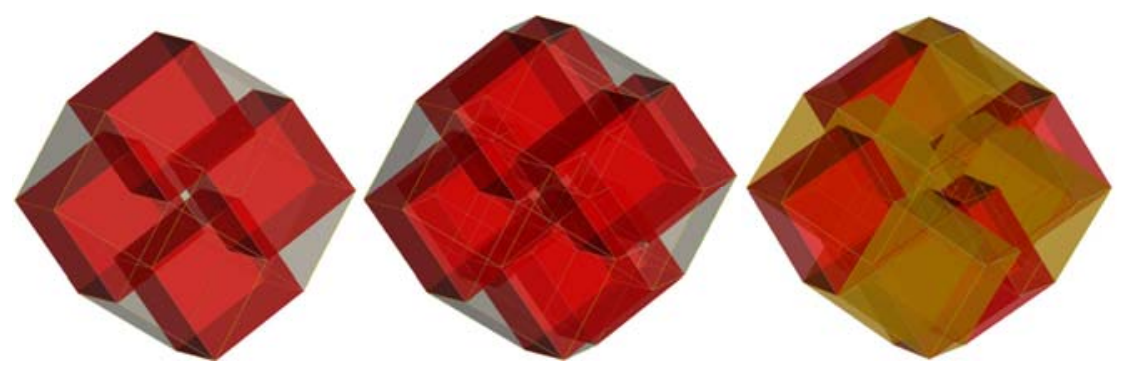

Fig. 10. Shift in direction of the third edge of the second cube: 5D-6D. The edges of former shifts are also shifted, so these define the new, yellow cubes

Choosing more and more differently oriented cubes, the above process can be continued. Hypercube 3-models can be constructed. All edges of these models join those of normal cubes. The amounts of elements used in the construction of models can be collected in a table. This can further help in a modeling program, following the above introduced steps that can be indicated by numbers in a table. The simplified Table I emphasizes the counting of the generated normal cubes during the process. 
Column 1: number of dimensions, column 2: initial shifted cubes, column 3: sum of the available cubes. These are elements only of a subset of differently shaped 3-models of 3-dimensional parts of the higher-dimensional cubes. The amounts of these are written in the last column of Table I. The gray cell shows that all edges of the considered 3model of the 15-cube join the edges of 20,480 normal cubes. The half turn of the initial arrangement of edges, showed in Fig. 2, right, results in a layout with tenfold rotational symmetry. It takes possible a very symmetric sequel of the modeling of hyper cubes, joining normal cubes, up to 30 dimensions and the process can be continued with more cubes oriented in different ways.

Table I

The simplified table emphasizes the counting of the normal cubes in between the models

\begin{tabular}{|c|c|c|c|c|}
\hline D & $\begin{array}{l}\text { cube }_{\text {is }} \\
2^{\text {D-3 }}\end{array}$ & $\begin{array}{c}\text { cube }_{\mathrm{ns}} \\
2^{\mathrm{D}-3} *(\mathrm{D}-3) / 3 \quad[(\mathrm{D}-3) / 3]\end{array}$ & \begin{tabular}{c}
\multicolumn{2}{c}{ cube } \\
$2^{\mathrm{D}-3} * \mathrm{D} / 3 \quad[\mathrm{D} / 3]$
\end{tabular} & $\begin{array}{c}\mathrm{j} 03 \\
2^{\mathrm{D}-3} \mathrm{C}_{\mathrm{D} ; 3}\end{array}$ \\
\hline 3 & 1 & 0 & 1 & 1 \\
\hline 4 & 2 & 0 & $2+0=2$ & 8 \\
\hline 5 & 4 & 0 & $4+20=4$ & 40 \\
\hline 6 & 8 & $2^{\mathrm{D}-3}+0 * 2^{3}=8$ & $8+8=16$ & 160 \\
\hline 7 & 16 & $8 * 2=16$ & $16+16=32$ & 560 \\
\hline 8 & 32 & $16 * 2=32$ & $32+32=64$ & 1792 \\
\hline 9 & 64 & $2^{\mathrm{D}-3}+8 * 2^{3}=128$ & $64+128=192$ & 5376 \\
\hline 10 & 128 & $128 * 2=256$ & $128+256=384$ & 15360 \\
\hline 11 & 256 & $256 * 2=512$ & $256+512=768$ & 42240 \\
\hline 12 & 512 & $2^{\mathrm{D}-3}+128 * 2^{3}=1536$ & $512+1536=2048$ & 112640 \\
\hline 13 & 1024 & $1536 * 2=3072$ & $1024+3072=4096$ & 292864 \\
\hline 14 & 2048 & $3072 * 2=6144$ & $2048+6144=8192$ & 745472 \\
\hline 15 & 4096 & $2^{\mathrm{D}-3}+1536 * 2^{3}=16384$ & $4096+16384=20480$ & 1863680 \\
\hline 18 & 32768 & 163840 & 196608 & 26738688 \\
\hline 21 & 262144 & 1572864 & 1835008 & 348651520 \\
\hline 24 & 2097152 & 14680064 & 16777216 & 4244635648 \\
\hline 27 & 16777216 & 134217728 & 150994944 & 49073356800 \\
\hline 30 & 134217728 & 1207959552 & 1342177280 & 544923975680 \\
\hline
\end{tabular}

\subsection{Modified models}

A topic of Experience Workshops is modeling of hyper cubes and connections to arts and mathematics [9], [10]. It requires a model with possibly less intersections of edges and few types of elements. The struts in a Zometool model of the 6-dimensional cube are parallel to edges of 2 cubes, out of the 5 ones, that can be constructed in the dodecahedron but the lengths of all second initial edges must be shortened to avoid the intersections (Fig. 11, left). This model was easier to follow visually by pupils of primary and secondary schools and by university students (Fig. 11, right). It is naturally explained by shadows, as axonometric projections, that a model does not have to have rectangular edge pairs. The creation of this model is based on shifted cubes in directions 
of the other 3 initial edges, also perpendicular to each other. The model edges join those of eight smaller and eight bigger normal cubes (Fig. 12).

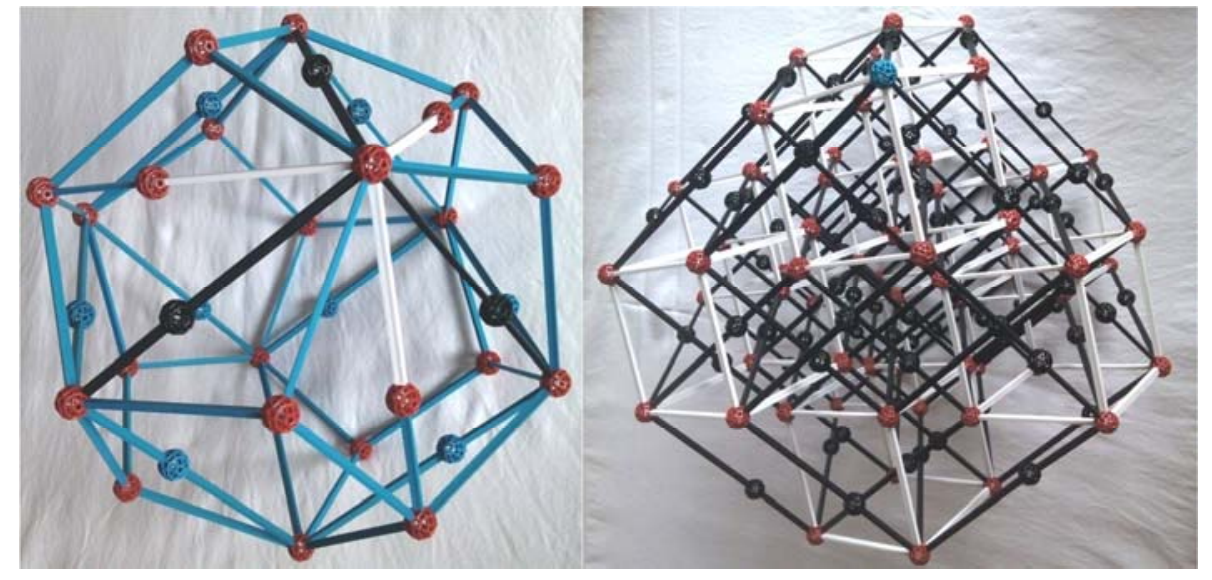

Fig. 11. Initial edges of the 3-model of the 6-cube and the model built with Zometool
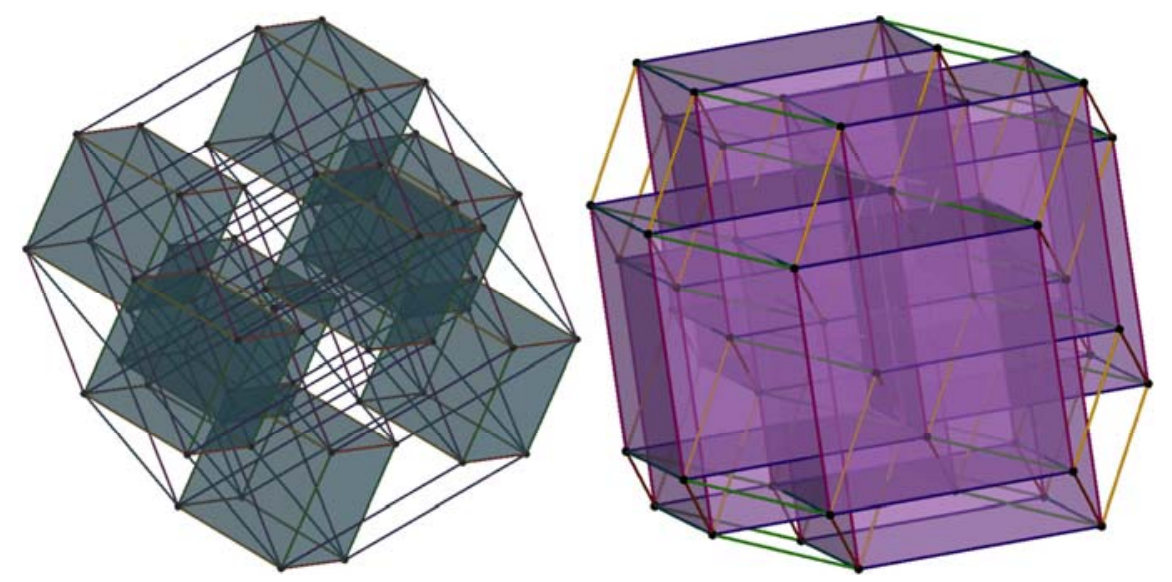

Fig. 12. The model edges join those of 8 smaller and 8 bigger normal cubes

Six pairs of edges overlap each other according to the golden mean. The common segments together with further 6 edges define 3 golden rectangles in a special disposition. The vertices of the rectangles can be linked by the edges of an icosahedron (Fig. 13, left). The lengths of the model struts and cube edges are proportioned in the golden mean. If the shorter cube edges are shortened 0.618 times again, then the struts of the new model have 12 intersection points (Fig. 13, right). The congruent parts of these models can be prefabricated by smaller groups of the workshop then they can collaborate in order to the construction of the next bigger parts (Fig. 14). 

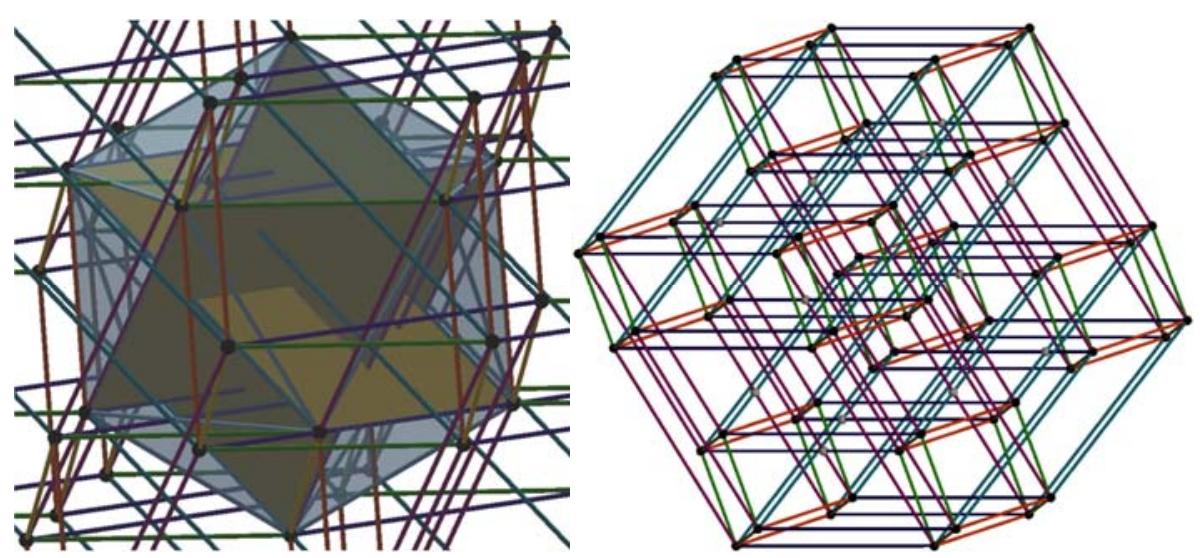

Fig. 13. The central part of the model together with the joining icosahedron and a variation of the above 3 -model of the 6-cube with 12 intersection points (indicated by gray spheres) of the edges

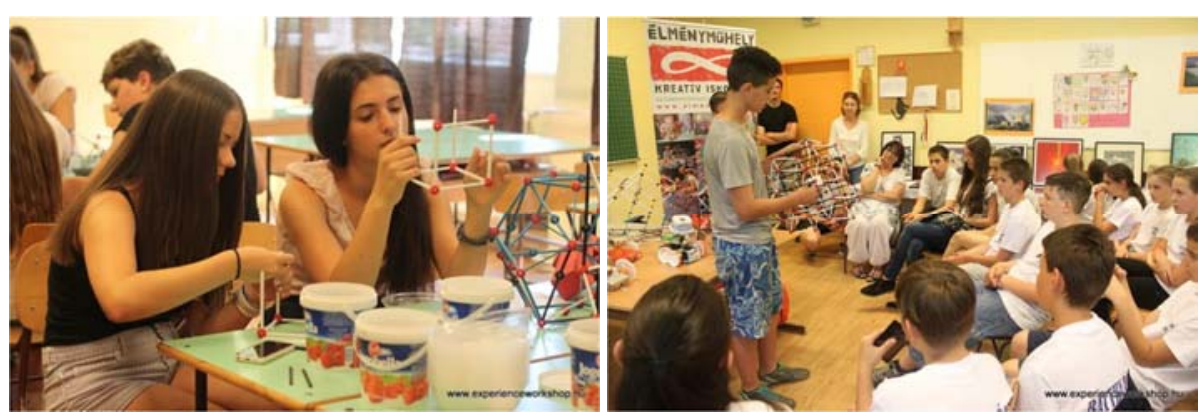

Fig. 14. Common work in pairs. Parts of the model can be prefabricated. Elected pupils report the activity to participants of other workshop groups (Photo: Judit Szanyi Nagy)

\section{Conclusion and remarks}

Former research topics in the field of more-dimensional cubes helped the work of ability management and workshop activities related to connections of arts and mathematics. The search for new and better usable models gave ideas to solve another research task. In this way, thea question of Author, posed at 2011 in a related paper could be answered. The truncated icosidodecahedron $(4,6,10)$ is one of the three Archimedean solids, which are zonotope 3-models of higher-dimensional cubes as well. The solid $(4,6,10)$ can also be the initial stone of a periodical space-filling mosaic if it is regarded as 3-model of the 15-cube and appropriately selected 3-models of the lowerdimensional parts are used as further stones.

The creation of the constructions and figures required for the research and the paper was aided by the AutoCAD program as well as by AutoLisp routines developed by the author. 


\section{Acknowledgements}

The elements of models used and built at workshops are provided by Zometool Inc.

This paper is dedicated to the memory of Professor Slavik Jablan who as mathematician, painter and real educator always presented deep and interesting connections among history, arts and mathematics in his lectures and papers as well as leading Experience Workshops.

\section{References}

[1] Vörös L. Two new space-filling mosaics based on a symmetric 3D model of the 10D cube, Pollack Periodica, Vol. 11, No. 1, 2016, pp. 81-90.

[2] Vörös L. Structures in the space of Platonic and Archimedean solids, Serbian Architectural Journal, Structural Systems, Vol. 3, No. 2, 2011, pp. 140-151.

[3] Vörös L. http://geometria.mik.pte.hu/videok.html, video 21.1-5 (last visited 3 July 2017).

[4] Coxeter H. S. M. Regular polytopes, 2nd ed, The MacMillan Company, New York, 1963.

[5] Towle R. Zonotopes, symmetrical-structures, 2008, http://zonotopia.blogspot.com (last visited 3 July 2017).

[6] Vörös L. Reguläre Körper und mehrdimensionale Würfel, KoG Scientific and Professional Journal of the Croatian Society for Geometry and Graphics, No. 9, 2005, pp. 21-27, http://master.grad.hr/hdgg/kog_stranica/kog9.pdf (last visited 3 July 2017).

[7] Grünbaum, B. Uniform tilings of 3-space, Geombinatorics, 1994, Vol. IV, No. 2, pp. 49-56.

[8] Vörös L. Specialties of models of the 6-dimensional cube, Proceedings of Bridges, 2010, Mathematics, Music, Art, Architecture, Culture, Pécs, Hungary, 24-28 July 2010, pp. 353-358.

[9] Experience Workshop, http://www.elmenymuhely.hu/?lang=en (last visited 3 July 2017).

[10] Fenyvesi K. Bridges: A world community for mathematical art, The Mathematical Intelligencer, Vol. 38, No. 2, 2016, pp. 35-45. 